\title{
From the index case to global spread: the global mobility based modelling of the COVID-19 pandemic implies higher infection rate and lower detection ratio than current estimates
}

\author{
Marian Siwiak $^{\text {Corresp., } 1}$, Pawel Szczesny ${ }^{1}$, Marlena Siwiak $^{1}$ \\ ${ }^{1}$ Data 3.0 Ltd, Sevenoaks, United Kingdom \\ Corresponding Author: Marian Siwiak \\ Email address: siwiak@data30.co.uk
}

\section{Background}

Since the outbreak of the COVID-19 pandemic, multiple efforts of modelling of the geo-temporal transmissibility of the virus have been undertaken, but none describes the pandemic spread at the global level. The aim of this research is to provide a high-resolution global model of the pandemic that overcomes the problem of biased country-level data on the number of infected cases. To achieve this we propose a novel SIR-type metapopulation transmission model and a set of analytically derived model parameters. We used them to perform a simulation of the disease spread with help of the Global Epidemic and Mobility (GLEAM) framework embedding actual population densities, commute patterns and long-range travel networks. The simulation starts on Nov $17^{\text {th }}, 2019$ with just a single presymptomatic, yet infectious, case in Wuhan, China, and results in an accurate prediction of the number of diagnosed cases after 154 days in multiple countries across five continents. In addition, the model outcome shows high compliance with the results of a random screening test conducted on pregnant women in the New York area.

\section{Methods}

We have built a modified SIR metapopulation transmission model and parameterized it analytically either by setting the values of the parameters based on the literature, or by assuming their plausible values. We compared our results with the number of diagnosed cases in twenty selected countries which provide reliable statistics but differ substantially in terms of strength and speed of undertaken Non-Drug Interventions. The obtained 95\% confidence intervals for the predictions are in agreement with the empirical data.

\section{Results}

The parameters that successfully model the pandemic are: the basic reproduction number $\boldsymbol{R}_{\boldsymbol{o}}, 4.4 ; \mathrm{a}$ latent non-infectious period of 1.1. days followed by 4.6 days of the presymptomatic infectious period; the probability of developing severe symptoms, 0.01 ; the probability of being diagnosed when presenting severe symptoms of 0.6 ; the probability of diagnosis for cases with mild symptoms or asymptomatic, 0.001 .

\section{Discussion}


Parameters that successfully reproduce the observed number of cases indicate that both $\boldsymbol{R}_{\boldsymbol{o}}$ and the prevalence of the virus might be underestimated. This is in concordance with the newest research on undocumented COVID-19 cases. Consequently, the actual mortality rate is putatively lower than estimated. Confirmation of the pandemic characteristic by further refinement of the model and screening tests is crucial for developing an effective strategy for the global epidemiological crisis. 
1 From the index case to global spread: The global

2 mobility based modelling of the COVID-19 pandemic 3 implies higher infection rate and lower detection ratio 4 than current estimates.

5 Marian Siwiak PhD 1, Pawel Szczesny PhD 1, Marlena Siwiak PhD 1

61 Data 3.0, Sevenoaks, TN14 7TG, UK

7 Corresponding Author:

8 Marian P. Siwiak PhD 1

93 Forge Way, Sevenoaks, TN14 7TG, United Kingdom

10 Email address: siwiak@data30.co.uk

\section{Abstract}

\section{Background}

Since the outbreak of the COVID-19 pandemic, multiple efforts of modelling of the geotemporal transmissibility of the virus have been undertaken, but none describes the pandemic spread at the global level. The aim of this research is to provide a high-resolution global model of the pandemic that overcomes the problem of biased country-level data on the number of infected cases. To achieve this we propose a novel SIR-type metapopulation transmission model and a set of analytically derived model parameters. We used them to perform a simulation of the disease spread with help of the Global Epidemic and Mobility (GLEAM) framework embedding actual population densities, commute patterns and long-range travel networks. The simulation starts on Nov $17^{\text {th }}, 2019$ with just a single pre-symptomatic, yet infectious, case in Wuhan, China, and results in an accurate prediction of the number of diagnosed cases after 154 days in multiple countries across five continents. In addition, the model outcome shows high compliance with the results of a random screening test conducted on pregnant women in the New York area.

\section{Methods}

We have built a modified SIR metapopulation transmission model and parameterized it analytically either by setting the values of the parameters based on the literature, or by assuming their plausible values. We compared our results with the number of diagnosed cases in twenty selected countries which provide reliable statistics but differ substantially in terms of strength and speed of undertaken Non-Drug Interventions. The obtained 95\% confidence intervals for the predictions are in agreement with the empirical data.

\section{Results}

The parameters that successfully model the pandemic are: the basic reproduction number $\boldsymbol{R}_{\boldsymbol{\theta}}, 4.4$; a latent non-infectious period of 1.1. days followed by 4.6 days of the presymptomatic infectious period; the probability of developing severe symptoms, 0.01 ; the probability of being diagnosed symptoms or asymptomatic, 0.001 . 


\section{Discussion}

39 Parameters that successfully reproduce the observed number of cases indicate that both $\boldsymbol{R}_{\boldsymbol{0}}$ and 40 the prevalence of the virus might be underestimated. This is in concordance with the newest 41 research on undocumented COVID-19 cases. Consequently, the actual mortality ratio is 42 putatively lower than estimated. Confirmation of the pandemic characteristic by further 43 refinement of the model and screening tests is crucial for developing an effective strategy for the 44 global epidemiological crisis.

\section{Introduction}

46 As of April 23 ${ }^{\text {rd }}$, 2020, novel coronavirus SARS-CoV-2 has already spread into 185 countries 47 and territories around the world (Dong, Du \& Gardner, 2020). With over two and half a million 48 confirmed infections and over 180 thousand deaths (Dong, Du \& Gardner, 2020), it became a 49 global challenge. COVID-19, the disease caused by this coronavirus, was characterised as a pandemic by WHO on March 11 th 2020.

While several different measures to contain the virus have been implemented by countries all over the world, their effectiveness remains to be seen. Until an effective treatment is available, the accuracy of the pandemic models and the decisions made on their basis are the major factors in reducing the overall mortality in the COVID-19 pandemic.

The models used to inform decision-makers differ significantly in their basic assumptions because it is the first coronavirus of such an impact in terms of the number of fatal cases. Moreover, the existing modelling approaches often use biased data for tuning parameters or assessing models' quality. In particular, most models use country-level data which is biased by one or many of the following factors: (i) level of transparency in acquiring, analysing, interpreting and reporting of data, (ii) level of detection effort, (iii) efficiency of introduced NonDrug Interventions, (iv) biased sampling of people to be tested (individuals showing severe and typical symptoms or suspected of having a contact with an infected person are more likely to be tested). The bias in data could be avoided by conducting well designed, random screening tests, but so far just a few such attempts have been made and they were limited to small and isolated communities in Italy (Lavezzo et al., 2020), and Germany (Streeck et al., 2020), or women admitted for delivery (Sutton et al., 2020).

Also, as of the date of submitting this work, there were no peer-reviewed geo-temporal models of the pandemic. We argue that creating a global model by fitting curves to observed data is impossible, unless the data used in this exercise is a result of large scale screening tests. As mentioned above, country-level data is biased and the nature of this bias is different for each country, mainly reflecting state policy towards the disease. Obviously, fitting model curves on global data bears a significant error, as this data is a mixture of all country-level biases.

Even the proper characteristic of the virus is hampered by the above-mentioned biases. For instance, early estimates of the basic reproduction number of the virus, $\boldsymbol{R}_{\boldsymbol{0}}$, were typically obtained using only Chinese data on the number of diagnosed cases (Zhang et al., 2020; Wu, Leung \& Leung, 2020; Liu et al., 2020). These estimates proposed the value of $\boldsymbol{R}_{\boldsymbol{0}}$ within the range 1.5-6.47, and the earliest most likely served as the basis for the January's official WHO estimate, which stated $\mathrm{R}_{0}=1.4-2.5$ ("Statement on the meeting of the International Health Regulations (2005) Emergency Committee regarding the outbreak of novel coronavirus 2019 (n$\mathrm{CoV}$ ) on 23 January 2020"). However, re-analysis of Chinese data provided an updated estimate of 5.7 (95\% CI 3.8-8.9) (Steven Sanche et al., 2020). 
82 Similar problems arise when estimating the actual prevalence of the virus. In this case, the

83 estimates are not only biased by the state policy, but also by the fact that many infections are 84 mild, asymptomatic or with atypical symptoms. In fact, many COVID-19 cases pass unnoticed, 85 for instance in Li's and coworkers China study, over 50\% (Li et al., 2020a).

86 The main objective of this research is to create a global model of the early stages of the 87 pandemic that would overcome the problem of the biased data on the number of infected cases. 88 This goal was achieved by creating a first global model of the COVID-19 pandemic that builds 89 on top of the successful modelling framework GLEAM (Van den Broeck et al., 2011). In contrast to many existing models, our attempt did not use biased country-level data on the number of infected cases to fit the model curve. Instead, it used a set of predefined parameters to simulate the spread of the disease around the world starting from the index case placed in Wuhan, China on Nov 17 $7^{\text {th }}, 2019$. The exact date was suggested by unverified press reports and used widely as a date of a disease contraction for "patient zero", but evolutionary tracing also suggests a similar timepoint ( $\mathrm{Li}$ et al., 2020b). The simulation took into account the current population densities all over the world, actual commute and flight networks, and travel ability of infected individuals. The simulation was run with 20 iterations for 154 days till Apr 19 19202. The obtained results were used to create $95 \%$ confidence intervals for curves of cumulative number of diagnosed cases, separately for each country in the world.

100 The presented model enables better understanding of the virus, its infectivity and detectability. Also, it may serve as a solid foundation for further attempts of global and country-level modelling. In particular, more detailed models that include information on introduced precautions may be created by making the detectability parameter variable in time and geographics. This would enable an optimal pandemic strategy to be established for each country.

\section{Materials \& Methods}

\section{Modelling software}

107 The model is based on the Global Epidemic and Mobility Model (GLEAM) framework (Balcan 108 et al., 2010), implemented in the GLEAMviz software (Van den Broeck et al., 2011). The 109 GLEAM model integrates sociodemographic and population mobility data in a spatially 110 structured stochastic disease approach to simulate the spread of epidemics at a worldwide scale. 111 It was previously used for a real-time numerical forecast of the global spreading of Influenza

112 A/H1N1 (Tizzoni et al., 2012), and the accuracy of that modelling was later confirmed (Tizzoni 113 et al., 2012).

\section{Data sources}

115 The reference data about the number of SARS-CoV-2 diagnosed patients in the period from Jan 116 22, 2020, to Apr 19 th, 2020 , were downloaded from the Johns Hopkins University of Medicine 117 Coronavirus Resource Center GitHub repository https://github.com/CSSEGISandData/COVID118 19. The provided data have been grouped by country. These data sources were used to assess the 119 quality of the model results. Empirical data for the time preceding Jan $22^{\text {nd }}, 2020$ is not available 120 in the cited source.

121 Information about the severity of developed symptoms was derived from the worldometer.info

122 website https://www.worldometers.info/coronavirus/. 
123 Information on testing efforts in selected countries, comes from the

124 https://ourworldindata.org/coronavirus-testing-source-data website.

125 Approximation of the number of mild and asymptomatic cases in the New York area was derived

126 from the results of a random screening test performed on women admitted for delivery at the

127 New York-Presbyterian Allen Hospital and Columbia University Irving Medical Center (Sutton

128 et al., 2020).

129 Information on introduced Non-Drug Interventions comes from publicly available sources.

130 Other data sources, such as subpopulation selection, commuting patterns, or air travel flows,

131 used during simulation, are embedded in the GLEAM software and well described by its

132 developers (Van den Broeck et al., 2011).

\section{Model parametrization}

134 Below and in Table 1, we present a set of parameters that was used in the model. Most 135 parameters were derived from the literature. In the absence of a reliable reference, the parameters 136 were assigned with the most plausible values by the authors based on the epidemiological 137 knowledge on SARS-CoV-2 and other viruses. The parameters' derivation method is 138 summarized in Table 1.

139 The average latency period ( $(\boldsymbol{p})$ ) of 5.6 days is a consensus of different estimations calculated 140 previously (Lauer et al., 2020). Due to 1) long $\boldsymbol{l} p$, effectively much longer than reported for other 141 coronaviruses, and 2) known cases of presymptomatic transmission (Woelfel et al., 2020; Tong 142 et al., 2020), for the modelling purposes we decided to split the latency period into two parts: 1) 143 average latent non-infectious period (Inip) of 1.1 days (based on the time of infectivity for other 144 viruses (Wallinga \& Teunis, 2004)), and 2) average presymptomatic infectious period (pip) of 1454.5 days. This split produces two parameters used in the model:

146 1) latency rate for the non-infectious period - non-infectious epsilon (niE):

$$
\text { nic }=1 / \text { lnip, }
$$

148 and

2) latency rate for the infectious period - latency rate infectious epsilon $(\boldsymbol{i} \varepsilon)$ :

$$
i \varepsilon=1 /(\operatorname{lp}-\ln i p) \text {. }
$$

As the Republic of Korea provided high quality, reliable data and conducted a large number of tests during the pandemic, we decided to use Korean proportion of "severe" to diagnosed cases as a base for the probability of developing the severe condition $(p S)$, and we set it to 0.01 . We assumed that patients with mild symptoms, in contrast to those in severe condition, are still capable of travelling. For model simplicity, we decided to merge into one compartment all mild and asymptomatic cases.

158 We decided to set the probability of detection of severe infection $(\boldsymbol{p D S})$ to 0.6 , in order to mimic two obstacles, typically preventing proper accurate diagnosis. Firstly, the majority of patients with a severe course of the disease are either chronically ill or above 60 (Zhou et al., 2020) their symptoms might be mistaken with those caused by their general health condition, and thus not reported on time. Secondly, the model is supposed to reflect the average illness detection around the globe, which includes many countries with low quality or underfinanced healthcare, where the number of SARS-CoV-2 tests is very limited. 
168 on previously defined $\boldsymbol{p S}$ and $\boldsymbol{p D S}$, as well as the ratio of total diagnosed to undiagnosed cases 169 (tDR):

$$
p D M=(t D R-p S * p D S) \div(1-p S) \text {. }
$$

171 Knowing the limitations of previous modelling attempts (Cowling et al.; Ganyani et al., 2020; Zhang et al., 2020; Chen et al., 2020; Wu, Leung \& Leung, 2020; Lin et al., 2020; Kucharski et al., 2020), we decided to test a radically different COVID-19 epidemiologic paradigm, i.e. a significantly lower $\boldsymbol{t D R}$. It means that in our model, we assume a higher proportion of undetected cases in comparison to other models proposed so far. Taking into account that none of them was capable of providing a plausible global simulation of the pandemic, plus the fact that the potentially low detectability has already been discussed in the literature (Nishiura et al., 2020; Li et al., 2020a; Day, 2020a,b; Kimball et al., 2020), we decided to test such a possibility in simulation by setting the lowest possible $t D R$. Its relation to $p D M$ sets its minimum to: $t D R>p S * p D S$.

181 For previously set $\boldsymbol{p} \boldsymbol{S}$ and $\boldsymbol{p D S}$ values, $\boldsymbol{t} \boldsymbol{D} \boldsymbol{R}$ must be greater than 0.006 , thus the value used in our simulation was set to 0.0061 . This value means that for 10,000 of COVID-19 cases only 61 are positively diagnosed. The justification for such a strong assumption is based on the following: (i) $\boldsymbol{t D R}$ reflects the average global detectability of the virus, including countries of low quality of public healthcare; (ii) $\boldsymbol{t D R}$ reflects the average detectability of the virus during the entire examined period of 154 days that describes early stages of the pandemic; (iii) the percentage of asymptomatic or atypical cases is currently unknown, but small-scale screening tests conducted so far indicate that even $88 \%$ of examined diagnosed cases could be asymptomatic (Sutton et al., 2020); and (iv) some of the currently used tests might be faulty e.g. when viral load is small (Pan et al., 2020).

Other important and interconnected parameters required by the model are as follows: the effective contact rate, $\boldsymbol{\beta}$; its reduction level for patients who developed severe symptoms of the disease but were not diagnosed, $\boldsymbol{r} \boldsymbol{\beta}$; and average recovery time since symptoms development $\boldsymbol{\mu}$. The parameter $\boldsymbol{\beta}$ is derived from the time a host remains infectious, $\boldsymbol{d}$, and the basic reproduction number of the virus, $\boldsymbol{R}_{\boldsymbol{\theta}}$ :

where:

$$
\beta=R_{0} \div d
$$

The estimation of $\boldsymbol{R}_{\boldsymbol{0}}$ is a topic widely discussed in the literature, with values ranging from 1.4 to 6.49 ("Statement on the second meeting of the International Health Regulations (2005) Emergency Committee regarding the outbreak of novel coronavirus (2019-nCoV)"; Majumder \& Mandl; Zhao et al.; Imai et al., 2020; Read et al., 2020; Liu et al., 2020). However, following the assumption of much higher than the currently suspected ratio of undiagnosed cases, we decided to use in our model a higher rate of transmissibility, yet well within the range of 2-5, modelled for SARS (Wallinga \& Teunis, 2004). The $\boldsymbol{R}_{\boldsymbol{\theta}}$ value assumed in our model is 4.4.

In our study $\boldsymbol{\mu}$ is derived from a safe quarantine period for diagnosed cases (Woelfel et al., 2020). As the safe quarantine time is estimated to be 10 days (Woelfel et al., 2020), we assumed $\boldsymbol{\mu}$ to last on average for 7 days from symptoms development to recovery. The sum of $\boldsymbol{\mu}$ and previously estimated pip (presymptomatic infectious period) results in $\boldsymbol{d}$ equal to 11.5 days, and $\boldsymbol{\beta}$ equal to 0.38261 . 
212 We decided to set $\boldsymbol{r} \boldsymbol{\beta}$ to 0.5 , following the assumption for this parameter used in GLEAM

213 modelling of the 2009 influenza outbreak (Balcan et al., 2010). Patients who were diagnosed

214 with COVID-19 are assumed isolated and as such, not spreading the disease any further.

\section{Model compartmentalization}

216 To model the virus spread, we modified the compartmental SIR metapopulation transmission model to represent the nature of the COVID-19 epidemic. In our model, we used seven different compartments (Figure 1).

219

220

221

222

223

224

225

226

227

228

229

230

231

232

233

234

235

236

237

238

239

240

241

242

243

244

245

1. Susceptible population - equal to the general global population. We assume no existing immunity to infection.

2. Latent non-infectious - infected population in the first incubation stage, not yet infectious.

3. Presymptomatic infectious - infected population already infectious, but without developed symptoms.

4. Mild symptoms - joint populations of asymptomatic cases and those with inconspicuous symptoms.

5. Severe symptoms - population infected by SARS-CoV-2 with symptoms affecting their travel ability.

6. Diagnosed - population identified as infected with the SARS-CoV-2 virus. This is the reference line for the model accuracy.

7. Recovered - joint populations of recovered and fatal cases. We assumed recovered individuals cannot be reinfected throughout the simulation, although the only evidence so far is for rhesus macaques (Ota, 2020) and WHO is still investigating the issue. It shall be emphasized, that this assumption may be invalidated in the future, and any further utilization of the presented model shall be accompanied by a thorough research into a current state of knowledge regarding the issue.

The last step was necessary to avoid the problem of unknown mortality ratio of the virus. It should be noted that the currently reported mortality ratio only applies to diagnosed cases (CFR, case fatality ratio), and its value still lacks consensus varying from $0.9 \%-2.1 \%$ (Wu et al., 2020). The true mortality ratio (IFR, infected fatality ratio) that takes into account all undiagnosed cases is likely to be much smaller. For China for instance, it has been estimated at $0.66 \%$ (Verity et al., 2020). However, even if we assume that we currently detect all COVID-19 cases, it should not exceed 3.4\% (WHO estimation) and this already is negligible from the perspective of our model (I.e. artificial increasing of the number of recovered, and thus immune individuals by this amount should not affect the model's final outcome much).

\section{Model running}

The prepared model served as an input for the GLEAM stochastic simulation. An initial simulation run with 10 iterations (the maximum allowed by publicly available free GLEAMviz Client application) was attempted prior to GLEAM development team licensing a one-time temporary increase in the number of public version iterations to 20. The GLEAM framework uses high-resolution worldwide population data, allowing for the definition of subpopulations according to a Voronoi decomposition of the world surface centered on the locations of International Air Transport Association (IATA)-indexed airports (www.iata.org). Short-range commuting networks for the defined subpopulations are constructed on the basis of data on the 
255

256

257

258

259

260

261

\section{2}

263

264

265

266

267

268

269

270

271

272

273

274

275

276

277

278

279

280

281

282

283

284

285

286

287

288

289

290

291

292

293

294

295

commuting patterns of 29 countries in five continents, generalized to a general gravity law for commuting flows, reproducing commuting patterns worldwide.

The stochastic simulation of the pandemic was started on Nov 17th, 2019. Although this date is stated only in non-academic sources, other reports also indicate mid-November as the time of the pandemic outbreak ( $\mathrm{Li}$ et al., 2020b). The simulation began with a single presymptomatic individual located in Wuhan, China, and the development of the pandemic spread was modelled for 154 days.

\section{Model results processing}

A single model run yields two sets of results. The first set is the median value and confidence intervals for the number of individuals per thousand which, at a given day, were moved to each of the compartments (presented in Figure 1). The second set is the median value and confidence intervals for a cumulative number of individuals per thousand, entering each of the compartments, until a given day. Both sets of results can be extracted with different resolutions globally, by hemisphere, continent, country, or the tessellated area surrounding IATA-registered airports.

For areas selected for detailed analyses of model results (i.e. twenty selected countries and the New York area), a cumulative number of transitions into a compartment of interest (i.e. "Diagnosed" and "Mild symptoms" respectively) was multiplied by the area population, and divided by thousand. The display of the model results was limited to the dates for which the experimentally derived data was available.

In order to compare model results with the random screening test from the New York area (Sutton et al., 2020), it was necessary to calculate the average number of undiagnosed mild and asymptomatic cases in this region $\left(\boldsymbol{c}_{s}\right)$ for the period covered by the experiment. The median and the lower and upper confidence limits of the number of individuals entering the "Mild symptoms" compartment at any day ( $\boldsymbol{n})$ of the simulation is provided by the GLEAM framework $\left(\boldsymbol{c}_{\boldsymbol{p}+\boldsymbol{m}}^{\boldsymbol{n}}, \boldsymbol{c}_{\boldsymbol{p}+\boldsymbol{l}}^{\boldsymbol{n}}\right.$, and $\boldsymbol{c}_{\boldsymbol{p}+\boldsymbol{u}}^{\boldsymbol{n}}$, respectively), while the number of individuals leaving the compartment $\left(\boldsymbol{c}_{\boldsymbol{p}-\text { - }}\right)$ was estimated similarly as in the framework:

$$
c_{p-}^{n}=c_{p}^{n} \div \mu
$$

where $c_{p}^{n}$ stands for the average number of infected but not diagnosed asymptomatic or mild symptoms cases at day $\boldsymbol{n}$. For each day ( $\boldsymbol{n})$ of the simulation, $\boldsymbol{c}_{\boldsymbol{p}}^{\boldsymbol{n}}$ and its lower and upper

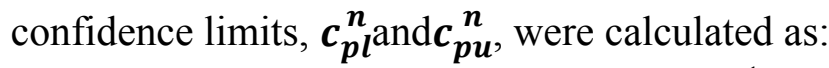

$$
\begin{gathered}
c_{p}^{n}=c_{p}^{n-1}+c_{p+m}^{n}-c_{p-1}^{n-1}, \\
c_{p l}^{n}=c_{p}^{n-1}+c_{p+l}^{n}-c_{p-1}^{n-1}, \\
c_{p u}^{n}=c_{p}^{n-1}+c_{p+u}-c_{p-1}^{n-1} .
\end{gathered}
$$

The lower and upper confidence limits for $\boldsymbol{c}_{\boldsymbol{s}}$, respectively $\boldsymbol{c}_{\boldsymbol{s}}$ and $\boldsymbol{c}_{\boldsymbol{s u}}$, used for comparison with confidence interval derived from the screening test, were obtained as:

$$
\begin{gathered}
c_{s l}=\left(\sum_{n=126}^{139} c_{p l}^{n}\right) /(139-126), \text { and } \\
c_{s u}=\left(\sum_{n=126}^{139} c_{p u}^{n}\right) /(139-126),
\end{gathered}
$$

where $\boldsymbol{n}=126$ and $\boldsymbol{n}=139$ stand for simulation steps referring to Mar 22 $2^{\text {nd }}, 2020$, and Apr $2^{\text {nd }}$, 2020, respectively.

The Excel workbook with performed calculations is provided as Supplementary Workbook 1. 


\section{Results}

297 The simulation modelled the pandemic spread for 154 days. The results for all subpopulations

298 around the globe are available in the shared model file (see the Data Sharing section below).

299 As overall data on the pandemic dynamics around the globe is likely to be biased by regions, 300 often considerable in size and population, for which official statistics might be inaccurate, we 301 decided not to compare overall model results with global data. Instead, we limited the analysis of results to twenty countries across five continents which are, in our belief: a) divergent in the proportion of the tested population (as reported in https://ourworldindata.org/coronavirus-testingsource-data), quality of healthcare, and strength of undertaken preventative measures; b) likely to provide the public with real data. We also compared the model outcome for the New York area subpopulation with the results obtained in a random screening test on women admitted for delivery (Sutton et al., 2020).

308 The obtained 95\% confidence intervals of predicted numbers of diagnosed patients were 309 compared with empirical data from twenty countries. In Figure 2, we present a percentage 310 difference over time between the number of reported confirmed cases and confidence interval 311 limits for modelled predictions. Positive values state that the model overestimates the number of 312 diagnosed cases; negative values indicate the underestimations of the model; for the observed numbers of diagnosed cases that are within the model's CIs the percentage difference is equal to 0 . For most of the selected countries, the model predictions fit well to the observed data, especially in the early stages of the pandemic.

316 Additionally, Figure 3 confronts the number of actual confirmed COVID-19 cases with 317 confidence intervals for the modelled number of diagnosed cases in twenty selected countries 318 from five continents for all 154 days of the simulation. Some countries present epidemic 319 dynamics different from the model. However, the direction of these deviations may be explained 320 by the measures overtaken by their governments, their societal response, or the number of tests carried per million of citizens. To show the possible influence of Non-Drug Interventions, we summarized them and marked the dates of their introduction in the country charts. Even though it is difficult to assess the effectiveness of precautions without detailed reports from the country in question, in some cases they seem to explain the observed discrepancies well. For instance the number of detected cases in Japan, Australia and New Zealand is much smaller than predicted by the model, which might be the result of the ban on flights from China introduced at the beginning of February combined with a geographical isolation of these countries. In contrast, most European countries started introducing preventive actions in March and the potential effects are only slightly visible in the last days of simulation. Similarly, the lack of concordance between model and empirical data in the case of the Republic of Korea may also be caused by the introduced precautions. However, in this case the preventive actions were of a different nature and are not shown in the country chart. Namely, the Republic of Korea introduced what was considered one of the largest and best-organised epidemic control programs in the world, consisting of measures to screen and isolate any infected people, as well as track and quarantine those who contacted them (South Korean Ministry of Health and Welfare).

Furthermore, the empirical data from the last day of the simulation, Apr 19 1 , 2020, was juxtaposed with the obtained confidence intervals in a single plot for all countries (Figure 4). In this plot the number of detected cases is presented as a percentage of the country population, in order to better show the width of the obtained confidence intervals in relation to the entire country population. From Figure 4 it is visible that model predictions are generally within the 
341 same order of magnitude as the observed data. So are the differences between upper and lower 342 confidence limits.

343 Finally, for the New York area and the time period between March $22^{\text {nd }}, 2020$ and April $2^{\text {nd }}$, 3442020 , we compared the model outcome with the results of a random screening test conducted on women admitted for delivery (Sutton et al., 2020). The cited experimental study revealed that out of 214 women tested, 33 were positively diagnosed with COVID-19, of which 4 had mild symptoms and 29 were asymptomatic. Assuming pregnant women are representative of the entire population and omitting severe COVID-19 cases from calculations (given patients in late pregnancy showing severe symptoms would have been admitted to the hospital earlier), the $95 \%$ CI for the true population proportion of mild or asymptomatic cases in the New York area, averaged over the examined period, is $0.11-0.21$. According to our model, in the same area and averaged over the same period, the $95 \%$ CI for this proportion is $0.19-0.23$. Although the predicted CI is not fully enclosed by the CI of the experimental result, their large overlap suggests high quality and accuracy of the model, started 139 days earlier from a single case on the other side of the globe.

Altogether, our 154-day long simulations of the pandemic seem to reflect the empirical data well. However, as is in the case of any model, this reflection is not perfect. The main reason for the discrepancies between model predictions and the reported number of COVID-19 cases is the fast governmental responses and early introduced precautions, which significantly influence the pace of the disease spread. Such preventive measures, for instance local flight bans, are not included in our simulations. In fact, the model depicts only the "natural" dynamics of the pandemic in the situation when governments do nothing to stop it. This means that in countries where overtaken actions were fast and effective, the model has a tendency to overestimate the number of detected cases.

The second potential reason for the observed discrepancies between model results and empirical data is the increase of the virus detectability in countries where the proportion of tested individuals is larger, leading to higher $\boldsymbol{t} \boldsymbol{D} \boldsymbol{R}$ than the one assumed in our model. To check this hypothesis, we calculated 95\% confidence intervals for the Spearman correlation coefficient between: a) the cumulative number of conducted tests per capita in a country, and b) the percentage difference between the cumulative number of detected cases and the lower or upper confidence limit of the CIs obtained in the model (i.e. if the model underestimates the number of detected cases, its upper confidence limit is used in calculations, and vice versa; if the observed data is within the predictions of the model, the difference is zero). The correlations' CIs were calculated separately for each day of the simulation, if only sufficient data on countries' testing effort were available in the datasource. Missing data on the number of carried tests were interpolated if possible. As some countries started testing earlier than others, the number of datapoints for correlations varies from 4 to 18 , depending on the date. The obtained CIs for correlations are plotted on a timeline in Figure 5. It is visible that within the used data and with a limited sample size of 4 to 18 countries, it is not possible to state the direction of the correlation and decide if the tested hypothesis is true.

We believe that further modelling efforts, that will include careful parameters' modifications over time in order to better reflect local responses to the pandemic, would greatly improve the accuracy of the simulations, but it is outside of the scope of this work. 
384 Data sharing

385 The model and the results of the simulation are freely available at

386 https://github.com/freesci/covid19.

387 The data used to generate Figure $\mathbf{5}$ is provided as Supplementary Table 1.

388 The data and calculations used to obtain confidence intervals for the proportion of the mild and

389 asymptomatic cases in the New York area is provided as Supplementary Workbook 1.

\section{Discussion}

To assess the quality of our model, its results should be compared to the observed data on the number of diagnosed cases, but this data suffers from four biases, listed previously. These biases cannot be totally eliminated, but careful selection of countries used in the analysis may reduce their impact significantly. In particular, we limited the analysis of model results to twenty countries which to our belief provide the most accurate and transparent reports on the number of infected cases (reducing bias $\mathrm{nr}$ 1). Selected countries are also divergent in terms of their detection efforts illustrated by the number of conducted tests per capita (reducing bias $\mathrm{nr} 2$ ). Additionally, our model depicts the early stages of the pandemic when Non-Drug Interventions were not yet introduced on a large scale in the selected countries, and if they were, the exact time of intervention was added to the final diagrams to show its impact on the number of diagnosed cases (reducing bias $\mathrm{nr} 3$ ). Furthermore, the proportion of symptomatic versus mild and asymptomatic cases is built in the model, so is the fact that symptomatic individuals are more likely to be tested (reducing bias $\mathrm{nr}$ 4). Finally, the detectability of the disease is also built in the model. This means that the presented confidence intervals depict the plausible range of diagnosed cases assuming a given detectability, not the actual number of infected individuals in the country. However, the latter may easily be assessed knowing the assumed detectability, or derived from the model file provided with this paper.

The presented model, due to its stochastic nature, avoids the problem of biased and inaccurate data and provides simulations of the pandemic spread for all the countries around the globe. It also has multiple implications concerning the major characteristics of the COVID-19 pandemic, such as the basic reproduction number of the virus $\boldsymbol{R}_{\boldsymbol{0}}$ (higher than previously assumed, yet not above the values estimated for other coronaviruses), and the average ratio of diagnosed cases $\boldsymbol{t D R}$ (much lower than assumed so far, especially for cases expressing mild symptoms and asymptomatic). Such a low $\boldsymbol{t} \boldsymbol{D} \boldsymbol{R}$ would indicate that the vast majority of the COVID-19 infections are so mild that they pass unnoticed. This is not implausible, considering the fact that there are 1.9 billion children aged below 15 years in the world (27\% of the global population) and predominantly (ca. 90\%) the course of their infections is mild or asymptomatic (Dong et al., 2020). Additionally, the $\boldsymbol{t} \boldsymbol{D R}$ used in our model indicates virus detectability averaged over the entire period of 154 days and over all countries in the world. Furthermore, some COVID-19 cases may show atypical symptoms (e.g. diarrhoea) (Gao, Chen \& Fang, 2020) which hinder correct diagnosis. Taking all this into account, plus the results of our model and latest reports on the low detectability of the virus (Nishiura et al., 2020; Li et al., 2020a; Day, 2020a,b; Kimball et al., 2020), one may risk a hypothesis that the virus is already more prevalent in the global population than shown in official statistics at the moment, and consequently, its mortality ratio is much lower.

To verify this hypothesis, further actions are required. At first, the model should be refined by stochastic fitting of parameters to the observed data. Also the sensitivity analysis of the 
428

429

430

431

432

433

434

435

436

437

438

439

440

441

442

443

444

445

446

447

448

449

450

451

452

453

454

455

456

457

458

459

460

461

462

463

464

465

466

467

parameters should be performed. Such a refined model could be used for the analysis of the effectiveness of the applied Non-Drug Interventions and possibly, for the modelling of future NDIs strategies. Secondly, a simulation with the $\boldsymbol{t} \boldsymbol{D} \boldsymbol{R}$ parameter increasing over time or diverging geographically might better reflect the actual virus detectability in the course of the pandemic. In this work, doubling the number of runs of simulation (from 10 to 20) did not influence the results in terms of their agreement with the observed number of cases. It allowed, however, an increase in the length of the simulation (from 135 to 154 days) while retaining the same order of magnitude of reported and predicted number of cases in each tested country. It means that in the case of extending the presented simulation further in time, it would be advised to introduce even a larger number of iterations to maintain the precision of predictions at the end of the simulation. Moreover, increasing the number of iterations would allow further narrowing of acquired CIs, increasing the model sensitivity to parameter values.

Finally, the real spread of the virus should be assessed empirically by conducting a sufficient number of tests on fully random samples as currently, most tests are limited to individuals with strong and typical symptoms. Only after obtaining a solid measurement of the actual prevalence of the virus, can one draw conclusions about its true mortality rate.

We emphasize that our conclusions are a hypothesis based on a single computational model and without empirical verification, but they serve as a platform for further research. At this stage, by no means should they be used as a reason for governmental decisions on lifting the precautions. Even if the true mortality of the virus is indeed lower than announced by the media, to our best knowledge many people remain in the high-risk group (e.g elderly, chronically ill, etc. (Baud et al., 2020)). Lack of population resistance facilitates their contact with the virus and may lead to a rapid increase of severe cases in a short time, as seen for example in Italy (Remuzzi \& Remuzzi, 2020), leading to the collapse of the healthcare system, which affects the entire society and results in many additional deaths not related to the virus itself. Careful use and tuning of NonDrug Interventions, constant balancing of the disease spread and healthcare capacity, protecting the most vulnerable individuals, farsighted anticipation and agility in decision making may altogether be able to minimize the number of deaths without resulting in the global economic breakdown.

\section{Conclusions}

Our model implies that the current consensus on the basic reproduction number of SARS-CoV-2 and its prevalence are misestimated. The overall global data on the pandemic dynamics seems strongly biased by large regions where official statistics may not reflect accurately the state of the epidemic, and by the fact that many COVID-19 cases may go unnoticed. The basic reproduction number of the virus should be confirmed based on reliable data, and its prevalence determined by conducting properly designed screening tests.

\section{References}

Balcan D, Gonçalves B, Hu H, Ramasco JJ, Colizza V, Vespignani A. 2010. Modeling the spatial spread of infectious diseases: the GLobal Epidemic and Mobility computational model. Journal of computational science 1:132-145.

Peer] reviewing PDF | (2020:03:47200:3:0:NEW 23 Jun 2020) 
468 Baud D, Qi X, Nielsen-Saines K, Musso D, Pomar L, Favre G. 2020. Real estimates of mortality

469 following COVID-19 infection. The Lancet infectious diseases. DOI: 10.1016/S1473-

$470 \quad 3099(20) 30195-X$.

471 Chen T-M, Rui J, Wang Q-P, Zhao Z-Y, Cui J-A, Yin L. 2020. A mathematical model for simulating the 472 phase-based transmissibility of a novel coronavirus. Infectious diseases of poverty 9:24.

473 Cowling BJ, Ali ST, Ng TWY, Tsang TK, Li JCM, Fong MW, Liao Q, Kwan MYW, Lee SL, Chiu SS, 474 Wu JT, Wu P, Leung GM. Impact assessment of non-pharmaceutical interventions against COVID47519 and influenza in Hong Kong: an observational study. DOI: 10.1101/2020.03.12.20034660.

476

477

478

479

480

481

482

483

484

485

486 487 488

Day M. 2020a. Covid-19: identifying and isolating asymptomatic people helped eliminate virus in Italian village. BMJ 368:m1165.

Day M. 2020b. Covid-19: four fifths of cases are asymptomatic, China figures indicate. BMJ 369:m1375.

Dong E, Du H, Gardner L. 2020. An interactive web-based dashboard to track COVID-19 in real time. The Lancet infectious diseases. DOI: 10.1016/S1473-3099(20)30120-1.

Dong Y, Mo X, Hu Y, Qi X, Jiang F, Jiang Z, Tong S. 2020. Epidemiological Characteristics of 2143 Pediatric Patients With 2019 Coronavirus Disease in China. Pediatrics. DOI: 10.1542/peds.20200702.

Ganyani T, Kremer C, Chen D, Torneri A, Faes C, Wallinga J, Hens N. 2020. Estimating the generation interval for COVID-19 based on symptom onset data. medRxiv.

Gao QY, Chen YX, Fang JY. 2020. 2019 Novel coronavirus infection and gastrointestinal tract. Journal of digestive diseases. DOI: 10.1111/1751-2980.12851.

Imai N, Cori A, Dorigatti I, Baguelin M, Donnelly CA, Riley S, Ferguson NM. 2020. Report 3: transmissibility of 2019-nCoV. Reference Source.

Kimball A, Hatfield KM, Arons M, James A, Taylor J, Spicer K, Bardossy AC, Oakley LP, Tanwar S, Chisty Z, Bell JM, Methner M, Harney J, Jacobs JR, Carlson CM, McLaughlin HP, Stone N, Clark S, Brostrom-Smith C, Page LC, Kay M, Lewis J, Russell D, Hiatt B, Gant J, Duchin JS, Clark TA, Honein MA, Reddy SC, Jernigan JA, Public Health - Seattle \& King County, CDC COVID-19 
494

495

496

497

498

499

500

501

502

503

504

505

506

507

508

509

510

511

512

513

514

515

516

517

518

519

Investigation Team. 2020. Asymptomatic and Presymptomatic SARS-CoV-2 Infections in Residents of a Long-Term Care Skilled Nursing Facility - King County, Washington, March 2020. MMWR. Morbidity and mortality weekly report 69:377-381.

Kucharski AJ, Russell TW, Diamond C, Liu Y, Edmunds J, Funk S, Eggo RM, Centre for Mathematical Modelling of Infectious Diseases COVID-19 working group. 2020. Early dynamics of transmission and control of COVID-19: a mathematical modelling study. The Lancet infectious diseases. DOI: 10.1016/S1473-3099(20)30144-4.

Lauer SA, Grantz KH, Bi Q, Jones FK, Zheng Q, Meredith HR, Azman AS, Reich NG, Lessler J. 2020. The Incubation Period of Coronavirus Disease 2019 (COVID-19) From Publicly Reported Confirmed Cases: Estimation and Application. Annals of internal medicine. DOI: 10.7326/M200504 .

Lavezzo E, Franchin E, Ciavarella C, Cuomo-Dannenburg G, Barzon L, Del Vecchio C, Rossi L, Manganelli R, Loregian A, Navarin N, Abate D, Sciro M, Merigliano S, Decanale E, Vanuzzo MC, Saluzzo F, Onelia F, Pacenti M, Parisi S, Carretta G, Donato D, Flor L, Cocchio S, Masi G, Sperduti A, Cattarino L, Salvador R, Gaythorpe KAM, Imperial College London COVID-19 Response Team, Brazzale AR, Toppo S, Trevisan M, Baldo V, Donnelly CA, Ferguson NM, Dorigatti I, Crisanti A. 2020. Suppression of COVID-19 outbreak in the municipality of Vo, Italy. medRxiv:2020.04.17.20053157.

Lin Q, Zhao S, Gao D, Lou Y, Yang S, Musa SS, Wang MH, Cai Y, Wang W, Yang L, He D. 2020. A conceptual model for the coronavirus disease 2019 (COVID-19) outbreak in Wuhan, China with individual reaction and governmental action. International journal of infectious diseases: IJID: official publication of the International Society for Infectious Diseases 93:211-216.

Li R, Pei S, Chen B, Song Y, Zhang T, Yang W, Shaman J. 2020a. Substantial undocumented infection facilitates the rapid dissemination of novel coronavirus (SARS-CoV2). Science. DOI: $10.1126 /$ science.abb3221.

Liu Y, Gayle AA, Wilder-Smith A, Rocklöv J. 2020. The reproductive number of COVID-19 is higher 
compared to SARS coronavirus. Journal of travel medicine 27. DOI: 10.1093/jtm/taaa021.

521 Li X, Zai J, Zhao Q, Nie Q, Li Y, Foley BT, Chaillon A. 2020b. Evolutionary history, potential

522 intermediate animal host, and cross-species analyses of SARS-CoV-2. Journal of medical virology.

523 DOI: $10.1002 / \mathrm{jmv} .25731$.

524 Majumder M, Mandl KD. Early Transmissibility Assessment of a Novel Coronavirus in Wuhan, China.

$525 \quad$ SSRN Electronic Journal. DOI: 10.2139/ssrn.3524675.

526 Nishiura H, Kobayashi T, Suzuki A, Jung S-M, Hayashi K, Kinoshita R, Yang Y, Yuan B,

527 Akhmetzhanov AR, Linton NM, Miyama T. 2020. Estimation of the asymptomatic ratio of novel

528 coronavirus infections (COVID-19). International journal of infectious diseases: IJID: official

529 publication of the International Society for Infectious Diseases. DOI: 10.1016/j.ijid.2020.03.020.

530 Ota M. 2020. Will we see protection or reinfection in COVID-19? Nature reviews. Immunology. DOI:

$531 \quad 10.1038 / \mathrm{s} 41577-020-0316-3$.

532 Pan Y, Long L, Zhang D, Yan T, Cui S, Yang P, Wang Q, Ren S. 2020. Potential false-negative nucleic

533 acid testing results for Severe Acute Respiratory Syndrome Coronavirus 2 from thermal inactivation

534 of samples with low viral loads. Clinical chemistry. DOI: 10.1093/clinchem/hvaa091.

535 Read JM, Bridgen JRE, Cummings DAT, Ho A, Jewell CP. 2020. Novel coronavirus 2019-nCoV: early

536 estimation of epidemiological parameters and epidemic predictions. medRxiv.

537 Remuzzi A, Remuzzi G. 2020. COVID-19 and Italy: what next? The Lancet 395:1225-1228.

538 South Korean Ministry of Health and Welfare.Coronavirus disease 19(COVID-19). Available at

539 http://ncov.mohw.go.kr/en/(accessed April 25, 2020).

540 Statement on the meeting of the International Health Regulations (2005) Emergency Committee regarding

541 the outbreak of novel coronavirus $2019(\mathrm{n}-\mathrm{CoV})$ on 23 January 2020. Available at

542 https://www.who.int/news-room/detail/23-01-2020-statement-on-the-meeting-of-the-international-

543 health-regulations-(2005)-emergency-committee-regarding-the-outbreak-of-novel-coronavirus-

544 (2019-ncov) (accessed April 23, 2020).

545 Statement on the second meeting of the International Health Regulations (2005) Emergency Committee

PeerJ reviewing PDF | (2020:03:47200:3:0:NEW 23 Jun 2020) 
546 regarding the outbreak of novel coronavirus (2019-nCoV). Available at https://www.who.int/news-

547 room/detail/30-01-2020-statement-on-the-second-meeting-of-the-international-health-regulations-

548 (2005)-emergency-committee-regarding-the-outbreak-of-novel-coronavirus-(2019-ncov) (accessed

$549 \quad$ March 20, 2020).

550 Steven Sanche, Yen Ting Lin, Chonggang Xu, Ethan Romero-Severson, Nick Hengartner, Ruian Ke.

5512020 . High Contagiousness and Rapid Spread of Severe Acute Respiratory Syndrome Coronavirus

552 2. Emerging Infectious Disease journal 26. DOI: 10.3201/eid2607.200282.

553 Streeck H, Hartmann G, Exner M, Schmid M. 2020. Preliminary result and conclusions of the COVID-19

554 case cluster study (Gangelt Municipality). Available at:

555 https://www.land.nrw/sites/default/files/asset/document/zwischenergebnis_covid19_case_study_gan

$556 \quad$ gelt_en.pdf(accessed April 29, 2020).

557 Sutton D, Fuchs K, D’Alton M, Goffman D. 2020. Universal Screening for SARS-CoV-2 in Women

558 Admitted for Delivery. The New England journal of medicine. DOI: 10.1056/NEJMc2009316.

559 Tizzoni M, Bajardi P, Poletto C, Ramasco JJ, Balcan D, Gonçalves B, Perra N, Colizza V, Vespignani A.

560 2012. Real-time numerical forecast of global epidemic spreading: case study of $2009 \mathrm{~A} / \mathrm{H} 1 \mathrm{~N} 1 \mathrm{pdm}$.

$561 \quad$ BMC medicine 10:165.

562 Tong Z-D, Tang A, Li K-F, Li P, Wang H-L, Yi J-P, Zhang Y-L, Yan J-B. 2020. Potential

563 Presymptomatic Transmission of SARS-CoV-2, Zhejiang Province, China, 2020. Emerging

$564 \quad$ infectious diseases 26. DOI: 10.3201/eid2605.200198.

565 Van den Broeck W, Gioannini C, Gonçalves B, Quaggiotto M, Colizza V, Vespignani A. 2011. The

566 GLEaMviz computational tool, a publicly available software to explore realistic epidemic spreading

$567 \quad$ scenarios at the global scale. BMC infectious diseases 11:37.

568 Verity R, Okell LC, Dorigatti I, Winskill P, Whittaker C, Imai N, Cuomo-Dannenburg G, Thompson H,

569 Walker P, Fu H, Dighe A, Griffin J, Cori A, Baguelin M, Bhatia S, Boonyasiri A, Cucunuba ZM,

570 Fitzjohn R, Gaythorpe KAM, Green W, Hamlet A, Hinsley W, Laydon D, Nedjati-Gilani G, Riley S,

571 van-Elsand S, Volz E, Wang H, Wang Y, Xi X, Donnelly C, Ghani A, Ferguson N. 2020. Estimates

Peer] reviewing PDF | (2020:03:47200:3:0:NEW 23 Jun 2020) 
of the severity of COVID-19 disease. medRxiv:2020.03.09.20033357.

573 Wallinga J, Teunis P. 2004. Different epidemic curves for severe acute respiratory syndrome reveal 574 similar impacts of control measures. American journal of epidemiology 160:509-516.

575 Woelfel R, Corman VM, Guggemos W, Seilmaier M, Zange S, Mueller MA, Niemeyer D, Vollmar P,

576 Rothe C, Hoelscher M, Others. 2020. Clinical presentation and virological assessment of

577 hospitalized cases of coronavirus disease 2019 in a travel-associated transmission cluster. medRxiv.

578 Wu JT, Leung K, Bushman M, Kishore N, Niehus R, de Salazar PM, Cowling BJ, Lipsitch M, Leung

579 GM. 2020. Estimating clinical severity of COVID-19 from the transmission dynamics in Wuhan,

$580 \quad$ China. Nature medicine 26:506-510.

581 Wu JT, Leung K, Leung GM. 2020. Nowcasting and forecasting the potential domestic and international

582 spread of the 2019-nCoV outbreak originating in Wuhan, China: a modelling study. The Lancet

$583 \quad 395: 689-697$.

584 Zhang S, Diao M, Yu W, Pei L, Lin Z, Chen D. 2020. Estimation of the reproductive number of novel

592 Zhou F, Yu T, Du R, Fan G, Liu Y, Liu Z, Xiang J, Wang Y, Song B, Gu X, Guan L, Wei Y, Li H, Wu X, coronavirus (COVID-19) and the probable outbreak size on the Diamond Princess cruise ship: A data-driven analysis. International journal of infectious diseases: IJID: official publication of the International Society for Infectious Diseases 93:201-204.

Zhao S, Lin Q, Ran J, Musa SS, Yang G, Wang W, Lou Y, Gao D, Yang L, He D, Wang MH. Preliminary estimation of the basic reproduction number of novel coronavirus (2019-nCoV) in China, from 2019 to 2020: A data-driven analysis in the early phase of the outbreak. DOI: 10.1101/2020.01.23.916395. Xu J, Tu S, Zhang Y, Chen H, Cao B. 2020. Clinical course and risk factors for mortality of adult inpatients with COVID-19 in Wuhan, China: a retrospective cohort study. The Lancet. DOI: 10.1016/S0140-6736(20)30566-3. 


\section{Figure 1}

Structure of compartments used in modelling.

A susceptible individual in contact with a person: 1) presymptomatic, 2) who developed mild symptoms, or 3) who developed severe symptoms, may contract the infection at rate $\boldsymbol{\beta}, \boldsymbol{\beta}$ or $\boldsymbol{r} \boldsymbol{\beta} * \boldsymbol{\beta}$, respectively, and enter the latent non-infectious compartment where he is infected but not yet infectious. During the non-infectious period, each individual has a probability of nic of becoming presymptomatic infectious. The presymptomatic cases have probability ic of developing severe or mild COVID-19 symptoms, with probabilities $\mathbf{p S}$ and $\mathbf{1 - p \boldsymbol { S }}$, respectively. The transition from both symptomatic groups occurs at $\boldsymbol{\mu}^{-1}$ rate. Individuals who developed severe symptoms do not travel within and between modelled subpopulations and may be either diagnosed with probability $\mathbf{p D S}$, or recover with probability of 1-pDS. Individuals whose mild (or non-existent) symptoms are not stopping them from traveling may be diagnosed with probability $\mathbf{P D M}$ or recover with probability 1-pDM. The diagnosed individuals are considered isolated and effectively non-contagious and recover with rate $\boldsymbol{\mu}^{-1}$. The recovery does not discriminate between true recovery and fatal cases. 


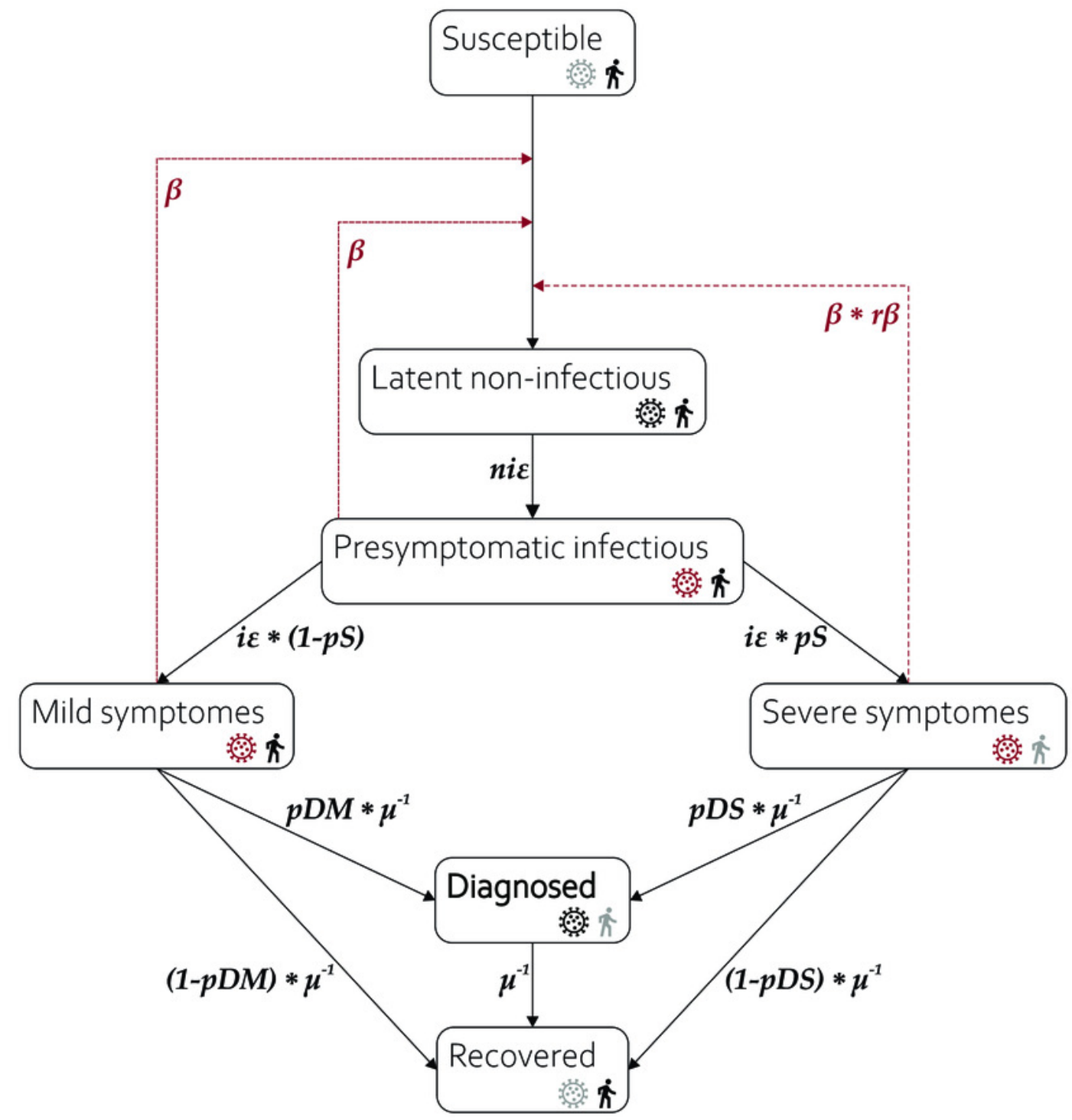
需: Uninfected
Infected
non-contagious
Infected
contagious

i Unrestricted

i mobility

Restricted 


\section{Figure 2}

Percentage difference over time between the number of reported cases and confidence intervals' limits for modelled predictions.

Positive values state that the model overestimates the number of diagnosed cases, negative values indicate the underestimations of the model. Observed numbers of cases that are within the model $\mathrm{Cls}$ are equal to 0 . For clarity, country plots were grouped by continents and presented in five plots: (A) Asia, (B) Europe, (C) North America, (D) South America, (E) Australia and Oceania. The large discrepancies for Japan, Australia, New Zealand and the Republic of Korea are putatively caused by the fast and pronounced reaction of their governments and early introduced NDIs which are not reflected in our model. 

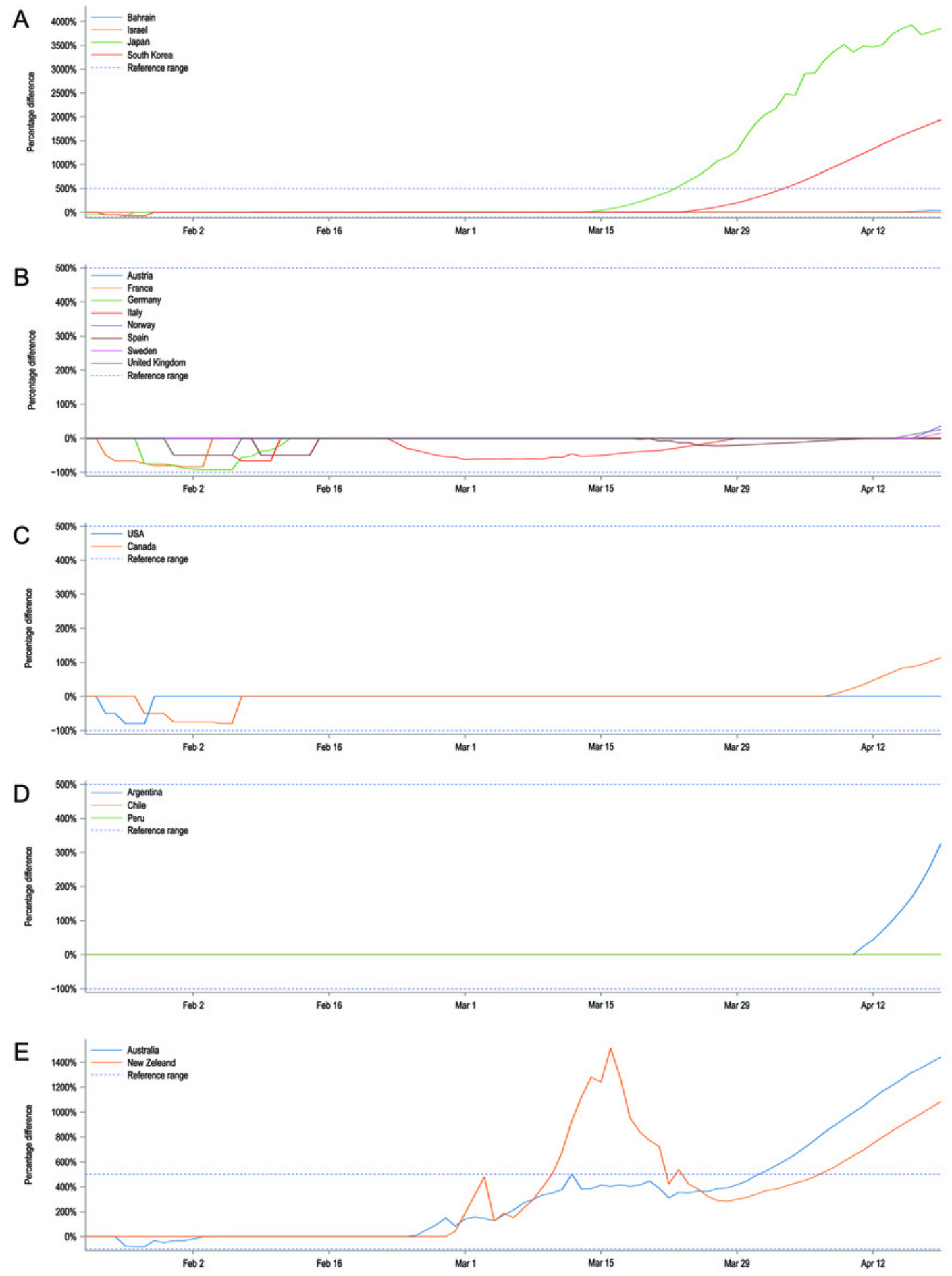


\section{Figure 3}

An overlay of modelled confidence intervals for the predicted cumulative number of diagnosed cases and the actual reported values shown for twenty selected countries.

The $y$-axes show the absolute number of diagnosed cases and due to different country populations are not unified. To facilitate comparisons a blue, dotted line was added as a reference indicating the $0.61 \%$ of the total population of a country. This value is the same as the tDR parameter used in our model reflecting the assumed ratio of detected to undetected cases. The confidence intervals obtained in our model will approach this value asymptotically. For most countries, observations agree well with model predictions. The observed discrepancies are most likely due to introduced NDIs which are not included in our model. The precautions were categorized and the dates of their introductions were marked on the plots with vertical lines, however, the assessment of their effectiveness is beyond the scope of this research. 

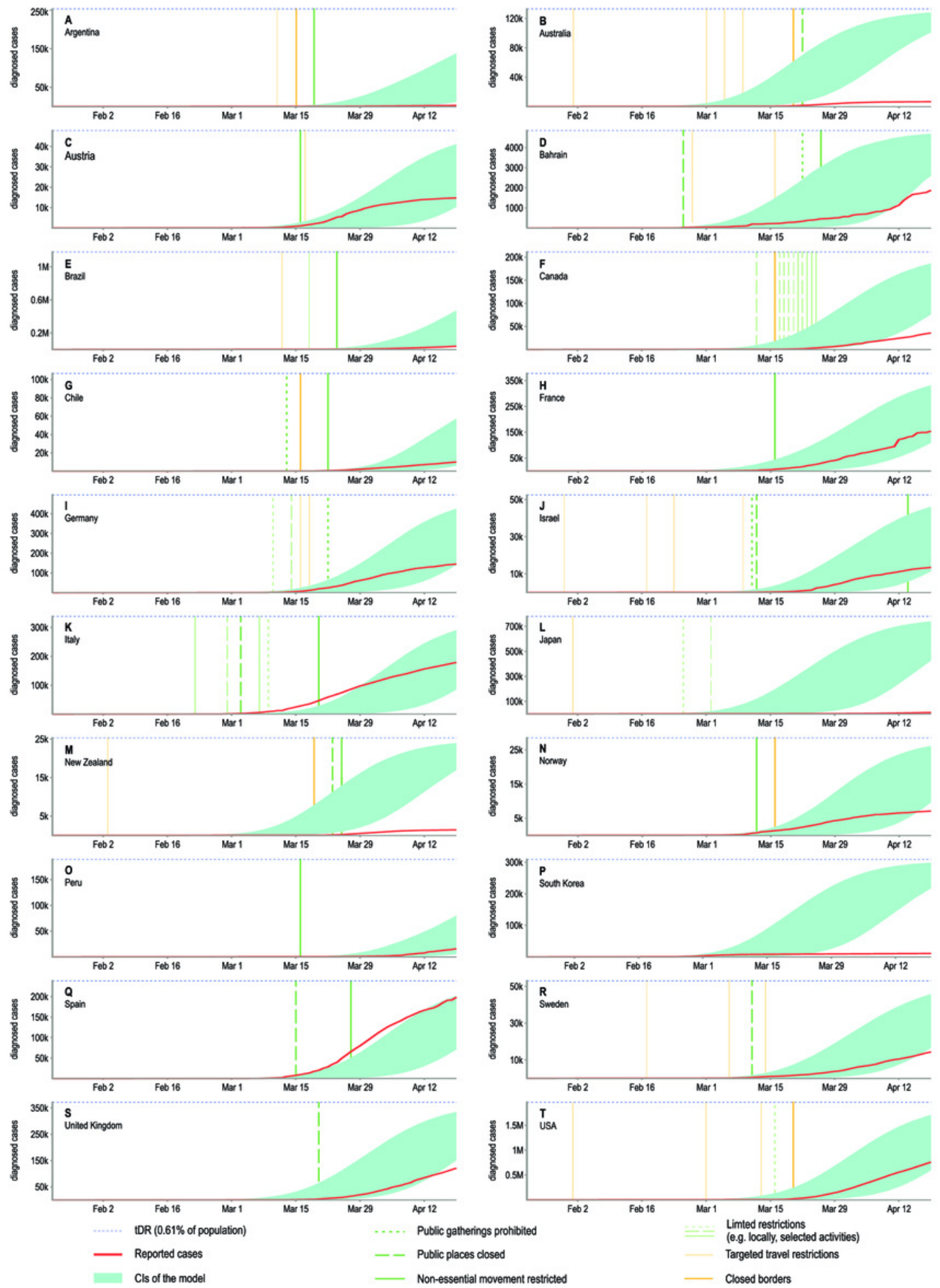


\section{Figure 4}

An overlay of modelled confidence intervals with the empirical data on the number of diagnosed cases for the last day of the simulation, Apr $19^{\text {th }}, 2020$.

The cumulative number of diagnosed cases is presented as a percentage of the country population, facilitating comparisons between countries. Model predictions are generally within the same order of magnitude as the observed data and obtained Cls are relatively narrow despite a limited number of iterations (20).

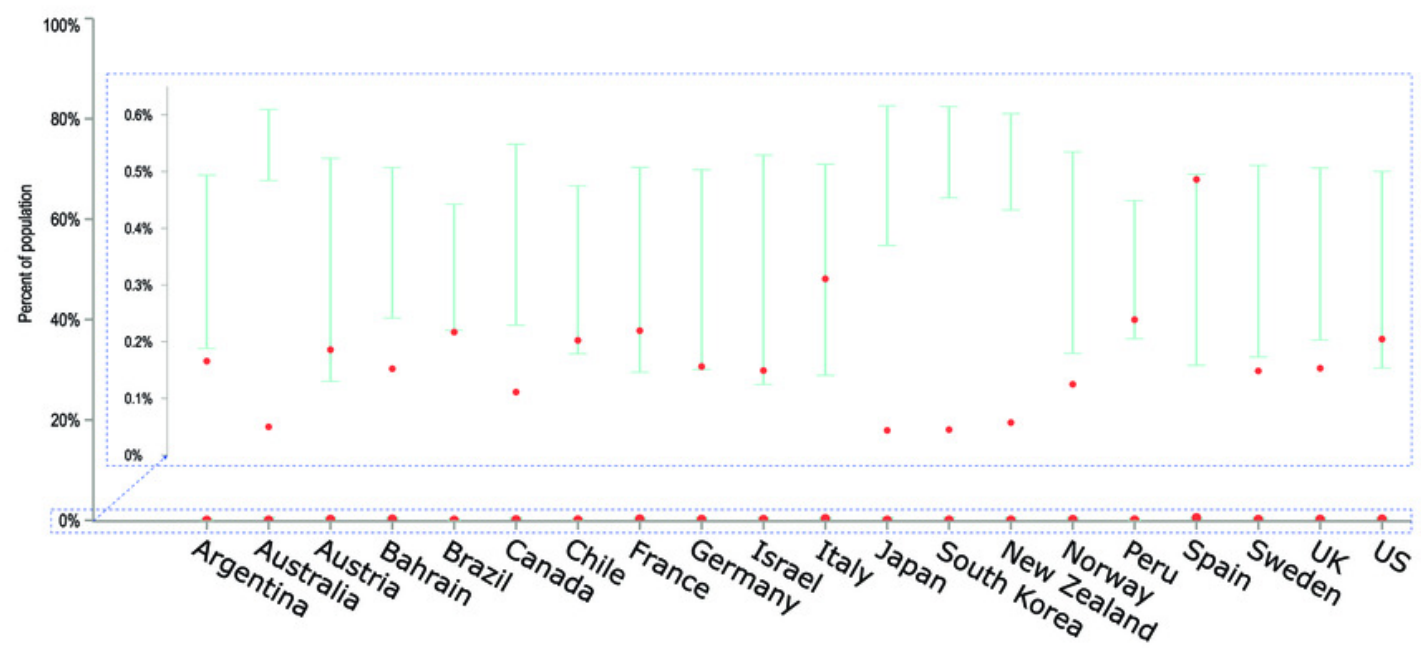




\section{Figure 5}

Correlation between presented model accuracy and per-country testing effort.

An overlay of $95 \%$ confidence intervals for the Spearman correlation coefficients between the interpolated, cumulative number of conducted tests per capita in a country, and the percentage difference between the actual number of detected cases and the lower or upper confidence limit of the Cls obtained in the model. The correlations were calculated separately for each day of the simulation if only sufficient data on testing effort is available. As some countries started testing earlier than others, the number of data points for correlations varies from 4 to 18, depending on the date. The width of the obtained Cls and the values of their limits indicate that with the avaialble data it is not possible to state the direction of the analyzed correlations.

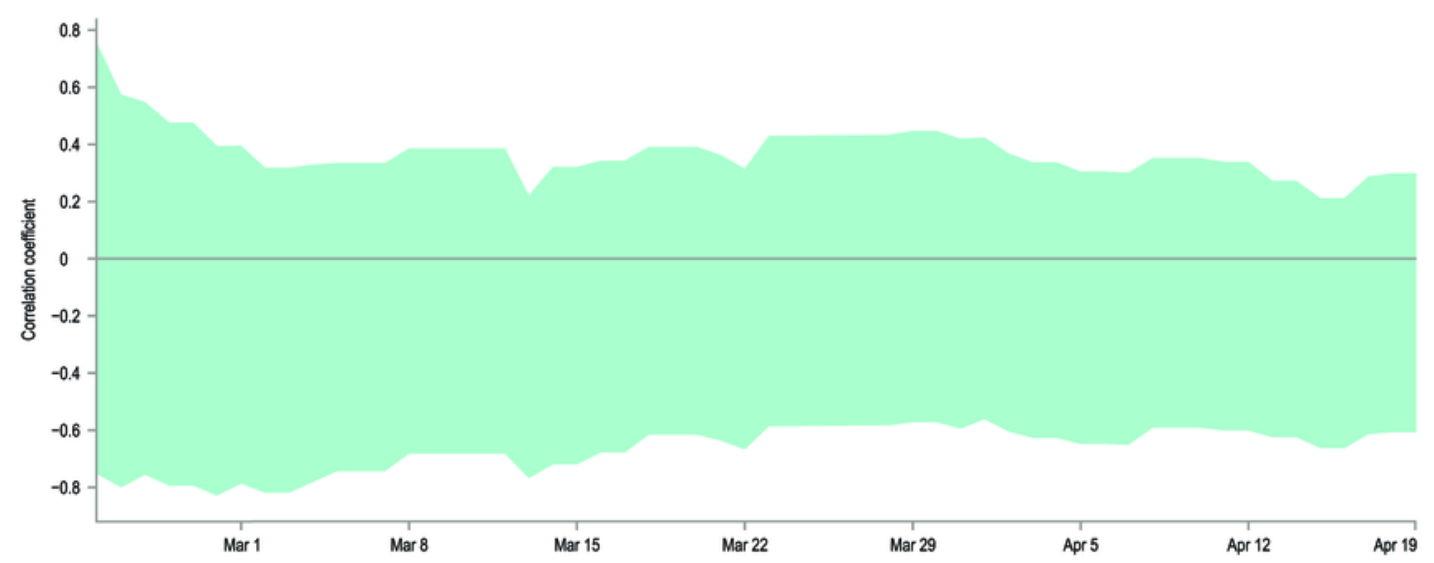




\section{Table 1 (on next page)}

Analytically derived model parameters 
1

\begin{tabular}{|c|c|c|c|}
\hline Parameter & Value & Description & Source/Derivation \\
\hline $\boldsymbol{R}_{0}$ & 4.4 & $\begin{array}{l}\text { Reproduction number for SARS- } \\
\text { CoV-2 }\end{array}$ & $\begin{array}{l}\text { Literature-based: assumed on the basis } \\
\text { of infectivity rates of other coronaviruses. }\end{array}$ \\
\hline$\mu$ & 7 days & $\begin{array}{l}\text { Average recovery time since } \\
\text { symptoms development }\end{array}$ & Literature-based \\
\hline$\beta$ & 0.38261 & Transmission rate & $\boldsymbol{\mu} \div \mathbf{R}_{\mathbf{0}}$ \\
\hline$r \beta$ & 0.5 & $\begin{array}{l}\text { Reduction in transmission rate } \\
\text { resulting from the undiagnosed } \\
\text { development of severe COVID-19 } \\
\text { symptoms }\end{array}$ & Literature-based \\
\hline Ip & 5.6 days & Average latency period & Literature-based \\
\hline Inip & 1.1 days & Average latent non-infectious period & $\begin{array}{l}\text { Literature-based: assumed on basis of } \\
\text { non-infectious period of Influenza A/H1N1 }\end{array}$ \\
\hline pip & 4.5 days & $\begin{array}{l}\text { Average presymptomatic infectious } \\
\text { period }\end{array}$ & Ip - Inip \\
\hline nic & $0.9(09)$ & $\begin{array}{l}\text { Probability of transition from Inip to } \\
\text { pip state }\end{array}$ & $1 \div$ Inip \\
\hline$i \varepsilon$ & $0.2(2)$ & $\begin{array}{l}\text { Probability of transition from } \\
\text { presymptomatic to symptomatic state }\end{array}$ & $1 \div(I p-$ Inip) \\
\hline$p S$ & 0.01 & $\begin{array}{l}\text { Probability of developing severe } \\
\text { COVID-19 symptoms }\end{array}$ & $\begin{array}{l}\text { Literature-data: The most often reported } \\
\text { ratio of severe to mild symptoms }\end{array}$ \\
\hline$p D S$ & 0.6 & $\begin{array}{l}\text { Probability of being diagnosed when } \\
\text { expressing severe COVID-19 } \\
\text { symptoms }\end{array}$ & $\begin{array}{l}\text { Assumed, taking into account that: } \\
\text { 1. In elderly patients, COVID may be } \\
\text { easily misdiagnosed, } \\
\text { 2. Most of the countries in the world do } \\
\text { not have sufficiently efficient healthcare } \\
\text { systems. }\end{array}$ \\
\hline$t D R$ & 0.0061 & $\begin{array}{l}\text { Rate of diagnosed SARS-CoV-2 } \\
\text { infected individuals }\end{array}$ & $\begin{array}{l}\text { Value representing the smallest possible } \\
\text { detectability }>p S * p D S\end{array}$ \\
\hline$p D M$ & $0.00(01)$ & $\begin{array}{l}\text { Probability of being diagnosed when } \\
\text { presenting mild or none COVID-19 } \\
\text { symptoms }\end{array}$ & $(t D R-p S * p D S) \div(1-p S)$ \\
\hline
\end{tabular}

